\title{
Hyper-parallel tempering Monte Carlo: Application to the Lennard-Jones fluid and the restricted primitive model
}

\author{
Qiliang Yan and Juan J. de Pablo \\ Department of Chemical Engineering, University of Wisconsin-Madison, Madison, Wisconsin 53706
}

(Received 14 July 1999; accepted 7 September 1999)

\begin{abstract}
A new generalized hyper-parallel tempering Monte Carlo simulation method is presented. The method is particularly useful for simulation of many-molecule complex systems, where rough energy landscapes and inherently long characteristic relaxation times can pose formidable obstacles to effective sampling of relevant regions of configuration space. In this paper, we demonstrate the effectiveness of the new method by implementing it in a grand canonical ensemble for the Lennard-Jones fluid and the restricted primitive model. Coexistence curves and critical behavior have been explored by the new method. Our numerical results indicate that the new algorithm can be orders of magnitude more efficient than previously available techniques. (C) 1999 American Institute of Physics. [S0021-9606(99)50945-8]
\end{abstract}

\section{INTRODUCTION}

Molecular simulations of complex systems are difficult, particularly at low temperatures, because configurations can get easily trapped in local energy minima, thereby precluding sampling of other, relevant regions of phase space. The inherently long characteristic relaxation times that often prevail in complex fluids further aggravate matters. Over the last few years several, powerful methods have been proposed to overcome the first difficulty; some examples are provided by multicanonical sampling, ${ }^{1,2} 1 / k$ sampling, ${ }^{3}$ simple tempering, ${ }^{4,5}$ expanded ensembles, ${ }^{6} \mathrm{~J}$-walking, ${ }^{7,8}$ and parallel tempering. ${ }^{9-11}$ While these methods are relatively effective at overcoming high-energy barriers, they do little to "accelerate" the slow relaxation of complex, many-molecule systems at low temperatures.

It has been increasingly recognized that open ensembles provide an effective means for overcoming slow-relaxation problems; molecules can get in and out of a system, thereby circumventing diffusional bottlenecks. In this paper we draw elements from simple and parallel tempering, J-walking, expanded ensembles, and histogram reweighting to propose a new, powerful Monte Carlo method for simulation of manymolecule systems. In doing so we combine the proven benefits of tempering or extended ensemble techniques with those of open-ensemble simulations. In this new method, configurations can hop simultaneously along several intensive variables. Furthermore, our formulation is well-suited for further combinations of expanded ensemble (or "simple tempering"') with parallel tempering. To distinguish our more general formulation from conventional (one-variable) parallel tempering, throughout this manuscript we refer to the new method as "hyper-parallel tempering Monte Carlo", (HPTMC). As shown in this manuscript, hyper-parallel tempering is significantly more efficient than existing methods for simulation of phase transitions. The new algorithm is relatively simple, and has the added benefit of being easily incorporated into molecular-dynamics or Monte Carlo simulation programs with minor modifications to existing codes.
In order to demonstrate the usefulness of HPTMC, in this paper, we have chosen to work with two systems: The Lennard-Jones fluid and the restricted primitive model of electrolyte solutions. On the one hand, the Lennard-Jones fluid has generally been adopted as a benchmark system on which to test new algorithms. Extensive studies of its phase behavior facilitate significantly comparisons to literature data and to existing algorithms. On the other hand, the restricted primitive model has attracted considerable interest over this past decade; its liquid-liquid phase transition at low temperatures has posed a challenge to theoreticians, and literature data have often been called into question. We use HPTMC to study that phase transition.

The paper is organized as follows. We begin by describing in detail how hyper-parallel tempering works. We then define the models and give a brief description of methodological technicalities used in our work, such as histogram reweighting and finite-size scaling techniques. We then present the results for the Lennard-Jones fluid and the restricted primitive model, and we compare these to available data. We conclude the paper by discussing the advantages and disadvantages of the new method, and by outlining some of its possible future applications.

\section{HYPER-PARALLEL TEMPERING MONTE CARLO}

For the sake of generality, we consider an arbitrary ensemble whose partition function is given by

$$
Z=\sum_{x} \Omega(x) w(x) \prod_{j} \exp \left(f_{j} q_{j}(x)\right),
$$

where $x$ denotes the state of the system, $\Omega(x)$ is the density of states, $w(x)$ is an arbitrary weighting function for state $x$, $f_{j}$ 's are generalized forces or potentials, and the $q_{j}$ 's are the corresponding conjugate generalized coordinates of the system. A grand canonical ensemble can thus be recovered by setting

$$
w(x)=\exp (-\beta U(x)), \quad f=\beta \mu, \quad q(x)=N(x),
$$


where $\beta=1 / k_{\mathrm{B}} T, T$ is temperature, $U(x)$ is the potential energy corresponding to configuration $x, \mu$ is the specified chemical potential, and $N(x)$ is the number of particles in configuration $x$. Alternatively, an isobaric-isothermal NPT ensemble can be recovered by setting

$$
w(x)=\exp (-\beta U(x)), \quad f=-\beta P, \quad q(x)=V(x),
$$

where $V(x)$ is the volume that corresponds to configuration $x$.

We now consider a composite ensemble consisting of $M$, noninteracting replicas of the above mentioned ensemble, each at a different set of generalized forces and weighting function. The complete state of the composite ensemble is specified through $\mathbf{x}=\left(x_{1}, x_{2}, \ldots, x_{M}\right)^{T}$, where $x_{i}$ denotes the state of the $i$ th replica. We define the partition function of the composite ensemble according to

$$
Z_{c}=\prod_{i=1}^{M} Z_{i}
$$

The un-normalized probability density of the composite state $\mathbf{x}$ is given by

$$
p(\mathbf{x})=\prod_{i=1}^{M} w_{i}\left(x_{i}\right) \prod_{j} \exp \left(f_{j, i} q_{j}\left(x_{i}\right)\right) .
$$

To sample configurations from the composite ensemble, a Markov chain is constructed in such a way as to asymptotically generate configurations according to the limiting distribution function appearing in Eq. (5). Two types of trial moves are used to realize that Markov chain:

(1) Standard Monte Carlo trial moves are used to locally update each of the replicas of the system. Since replicas do not interact with each other, standard Metropolis acceptance-rejection criteria (for the underlying ensemble) are employed within each replica.

(2) Configuration swaps are proposed between pairs of replicas $i$ and $i+1$, so that

$$
\begin{aligned}
& x_{i}^{\text {new }}=x_{i+1}^{\text {old }}, \\
& x_{i+1}^{\text {new }}=x_{i}^{\text {old }} .
\end{aligned}
$$

To enforce a detailed-balance condition, the pair of replicas to be swapped is selected at random, and the trial swap is accepted with probability:

$$
\begin{aligned}
p_{\text {acc }}\left(x_{i} \leftrightarrow x_{i+1}\right)= & \min \left[1, \frac{w_{i}\left(x_{i+1}\right) w_{i+1}\left(x_{i}\right)}{w_{i}\left(x_{i}\right) w_{i+1}\left(x_{i+1}\right)}\right. \\
& \left.\times \prod_{j} \exp \left(-\Delta f_{j} \Delta q_{j}\right)\right],
\end{aligned}
$$

where $\Delta f_{j}=f_{j, i+1}-f_{j, i}$ is the difference in generalized force $f_{j}$ between the two replicas, and $\Delta q_{j}=q_{j}\left(x_{i+1}\right)-q_{j}\left(x_{i}\right)$ is the difference of the corresponding conjugate generalized coordinates.

So far tempering has been discussed for the case in which several fields are sampled at the same time. A further generalization is to now couple the technique to that of expanded ensembles (or "simple tempering"), so one can simulate long polymer chains at high densities more efficiently. In this latter generalization, the whole simulation system consists of several expanded grand canonical ensembles. Each replica includes a tagged chain, whose length fluctuates during the simulation. When a swap trial move is attempted, these tagged chains are also switched. The corresponding acceptance criterion can be obtained by following a development analogous to the one presented above. More details of the generalization and its application are given elsewhere. ${ }^{27}$

\section{MODELS AND SIMULATION METHODS}

\section{A. Models}

In this work, we apply the hyper-parallel tempering method to two different molecular models. The first is the Lennard-Jones fluid. The potential energy between a pair of particle $i$ and $j$, separated by a distance $r_{i j}$, is defined by

$$
U_{i j}=4 \epsilon\left[\left(\frac{\sigma}{r_{i j}}\right)^{12}-\left(\frac{\sigma}{r_{i j}}\right)^{6}\right],
$$

where $\epsilon$ is the depth of the attractive well, and $\sigma$ is a parameter that controls the size of the particles. In this work, the Lennard-Jones potential-energy function is truncated at a cutoff distance $r_{c}$. To be consistent with previous studies, we choose $r_{c}=2.5 \sigma$, and the potential is left unshifted.

The restricted primitive model (RPM) of an ionic solution consists of $2 N$ hard spheres of diameter $\sigma$, half of them carrying a negative charge and the other half carrying a positive charge. A solvent is not modeled explicitly; instead, it is simply considered as a dielectric continuum with dielectric constant $D$. The charged spheres interact via a Coulomb potential,

$$
U_{i j}=\left\{\begin{array}{l}
+\infty, \quad r_{i j} \geqslant \sigma \\
\frac{e^{2}}{4 \pi D D_{0}} \frac{z_{i} z_{j}}{r_{i j}}, \quad r_{i j}<\sigma
\end{array}\right.
$$

where $z_{i} e$ and $z_{j} e$ are the charges carried by ions $i$ and $j$, respectively, $e$ is the charge of the electron $e=1.602$ $\times 10^{-19} \mathrm{C}$, and $D_{0}$ is the dielectric permeability of vacuum, $D_{0}=8.85 \times 10^{-12} \mathrm{C}^{2} \mathrm{~N}^{-1} \mathrm{~m}^{-2}$. Note that the restricted primitive model requires that $\left|z_{i}\right|=\left|z_{j}\right|$.

Throughout this work results are reported in reduced units. For the Lennard-Jones fluid, the reduced temperature is defined as $T^{*}=k_{\mathrm{B}} T / \epsilon$, where $k_{\mathrm{B}}$ is Boltzmann's constant. The reduced density is defined as $\rho^{*}=N \sigma^{3} / V$, with $N$ being the number of particles and $V$ the volume of the simulation box. For the restricted primitive model, the reduced temperature is defined as $T^{*}=4 \pi D D_{0} \sigma k_{\mathrm{B}} T / e^{2}$, and the reduced density is given by $\rho^{*}=2 N \sigma^{3} / V$.

\section{B. Simulation details}

In this work, the hyper-parallel tempering method is implemented in the grand canonical ensemble. By substituting Eq. (2) into Eq. (7), we arrive at the following acceptance criteria for swapping two replicas:

$$
p_{\text {acc }}\left(x_{i} \leftrightarrow x_{i+1}\right)=\min [1, \exp (\Delta \beta \Delta U-\Delta(\beta \mu) \Delta N)]
$$


where $\quad \Delta \beta=\beta_{i+1}-\beta_{i}, \quad \Delta U=U\left(x_{i+1}\right)-U\left(x_{i}\right), \quad \Delta(\beta \mu)$ $=\beta_{i+1} \mu_{i+1}-\beta_{i} \mu_{i}$, and $\Delta N=N\left(x_{i+1}\right)-N\left(x_{i}\right)$.

Two kinds of trial moves are employed, namely, trial displacements of particles and trial creations or destructions of particles. The relative frequency of these two kinds of trial moves is set to be unity. For the restricted primitive model, the conventional Ewald sum method is used to calculate long-range contributions to the energy arising from the slowdecaying Coulomb potential. Conducting boundary conditions are employed in our calculations; It has been pointed out in the literature that such a boundary condition is essential for simulations of ionic systems. ${ }^{20,21}$ Particle creations and destructions are conducted in pairs to preserve electroneutrality. A distance-bias Monte Carlo scheme is also employed to further facilitate particle transfer moves. ${ }^{23}$

During the simulation, the number of particles $N$ and the total potential-energy $U$ of each replica is recorded. The joint distributions $p(N, U)$ are accumulated in the form of a histogram. Note that for the restricted primitive model, $N$ is the number of particle pairs (the chemical potential is defined as the total chemical potential per ion pair).

\section{Histogram reweighting technique}

The histogram reweighting technique ${ }^{12-14}$ is designed to extract a maximal amount of information from the results of a set of molecular simulations. It has been widely used to determine the near-critical and sub-critical coexistence properties of fluids. For completeness, in this work we provide a brief description of its implementation; for additional details, readers are referred to the original publications. In a grand canonical simulation, the relevant thermodynamic variables are the temperature $T$ and the chemical potential $\mu$. A simple grand canonical simulation can be conducted at $T=T_{0}$ and $\mu=\mu_{0}$, and a two-dimensional histogram of energy and number of molecules can be constructed. The entries to such a histogram $H(N, U)$ represent the number of times that the system is observed with $N$ particles and potential-energy $U$. If the total number of realizations of the system is denoted by $K$, the probability $P\left(N, U, T_{0}, \mu_{0}\right)$ that the system has $N$ particles and energy $U$ at $T_{0}$ and $\mu_{0}$ is given by

$$
P\left(N, U, T_{0}, \mu_{0}\right)=H(N, U) / K .
$$

The probability distribution for a grand canonical ensemble is given by

$$
P(N, U, T, \mu)=\frac{\Omega(N, V, U) \exp (-\beta U+N \beta \mu)}{\Xi(\mu, V, T)},
$$

where $\Omega(N, V, U)$ is the microcanonical partition function (density of states at $N$ and $U$ ), and $\Xi$ is the grand partition function, given by

$$
\Xi(\mu, V, T)=\sum_{N} \sum_{U} \Omega(N, V, U) \exp (-\beta U+N \beta \mu) .
$$

Combination of Eqs. (11) and (12) provides a Monte Carlo estimate of $\Omega(N, V, U)$, given by

$$
\Omega(N, V, U)=w H(N, U) \exp \left(\beta_{0} U-N \beta_{0} \mu_{0}\right),
$$

where $w=\Xi(\mu, V, T) / K$ is a proportionality constant. Because $\Omega(N, V, U)$ is independent of $T$ and $\mu$, the probability that the system has $N$ particles and energy $U$ at a different state point $T$ and $\mu$ can be estimated according to

$$
\begin{aligned}
& P(N, U, T, \mu) \\
& \quad=\frac{H(N, U) \exp \left[-\left(\beta-\beta_{0}\right) U+N\left(\beta \mu-\beta_{0} \mu_{0}\right)\right]}{\Sigma_{N} \Sigma_{U} H(N, U) \exp \left[-\left(\beta-\beta_{0}\right) U+N\left(\beta \mu-\beta_{0} \mu_{0}\right)\right]} .
\end{aligned}
$$

The average value of any function of $N$ and $U$ can therefore be estimated from

$$
\langle A\rangle=\sum_{N} \sum_{U} A(N, U) P(N, U, T, \mu) .
$$

In practice, the extrapolation scheme provided by Eq. 15 is only useful if the distance between $\left(T_{0}, \mu_{p}\right)$ and $(T, \mu)$ is not too large. The range of energy and number of particles covered by a single histogram is limited, and extrapolations beyond that range are unfounded.

In order to expand the applicability of the histogram reweighting technique, several simulations can be carried out at different state points. Such simulations have traditionally been conducted independently of each other, i.e., in series. In this work, such simulations are conducted in parallel according to our tempering scheme; each replica corresponds to a different state point, and the resulting histograms are combined to generate thermodynamic predictions over a wide range of conditions.

The underlying bridge to combine multiple histograms is the fact that the microcanonical partition function $\Omega(N, V, U)$ is independent of temperature and chemical potential. Estimates of $\Omega(N, V, U)$ by Eq. (14) from different simulations should be consistent with each other (within the statistical uncertainty of the calculations). If histograms from different runs overlap sufficiently, it is possible to find a set of proportionality constants $\{w\}$ so that this requirement is fulfilled. An optimal estimate of $\Omega(N, V, U)$ can then be determined as a weighted average of $\Omega$ 's extracted from different runs. Ferrenberg and Swendsen proposed an efficient method to determine the sought-after proportionality constants and the weighting factors: for $R$ simulation runs, the optimal (unnormalized) probability distribution is given by

$$
P(N, U ; \mu, \beta)=\frac{\sum_{n=1}^{R} H_{n}(N, U) \exp (-\beta U+N \beta \mu)}{\sum_{m=1}^{R} K_{m} \exp \left(-\beta_{m} U+N \beta_{m} \mu_{m}-C_{m}\right)},
$$

where $K_{m}$ is the total number of realizations in run $m$. The constants $C_{m}$ must be determined self-consistently by the iterative relationship

$$
\exp \left(C_{m}\right)=\sum_{N} \sum_{U} P\left(N, U ; \mu_{m}, \beta_{m}\right) .
$$

In this work, we use a quasi-Newton method to solve the nonlinear equations [Eqs. (17) and (18)].

At sub-critical temperatures, the grand canonical density distribution is characterized by a double peaked structure, provided the chemical potential is close to the coexistence 
value. If the so-called "two-state" approximation is adopted, ${ }^{19}$ the criteria for phase equilibrium are equivalent to requiring that the areas under the two peaks be equal. The determination of the precise location of a coexistence point can, therefore, be achieved by simply tuning the chemical potential at any given temperature, until the areas under the two peaks become the same; the coexisting densities of the two coexisting phases then correspond to the mean densities under these two peaks.

\section{Mixed-field finite-size scaling}

The periodic boundary conditions generally employed in molecular simulations suppress long-range fluctuations; near a critical point, thermodynamic properties calculated by simulation are, therefore, different from those corresponding to the thermodynamic limit. However, it is possible to estimate with high accuracy the coordinates of the critical point by resorting to finite-size scaling techniques. ${ }^{15,16}$

For the systems studied here, the relevant scaling fields consist of combinations of the temperature and chemical potential and are given by

$$
\begin{aligned}
& \tau=\beta_{c}-\beta+s\left(\mu-\mu_{c}\right), \\
& h=\mu-\mu_{c}+r\left(\beta_{c}-\beta\right),
\end{aligned}
$$

where $\tau$ is the thermal scaling field, $h$ is the ordering scaling field, and subscript $c$ serves to denote a quantity evaluated at the critical point. Parameters $s$ and $r$, which control the degree or extent of field mixing, are system specific.

Conjugate to the two scaling fields are two scaling operators, namely, the ordering operator $\mathcal{M}$ and an energy-like operator $\mathcal{E}$. They consist of linear combinations of the particle density $\rho$ and the energy density $u=U / V$

$$
\begin{aligned}
& \mathcal{M}=\frac{1}{1-s r}(\rho-s u), \\
& \mathcal{E}=\frac{1}{1-s r}(u-r \rho) .
\end{aligned}
$$

For the simple case of models with Ising symmetry (where $s=r=0), \mathcal{M}$ is simply the magnetization and $\mathcal{E}$ is just the energy density.

In the critical region, the probability distributions of $\mathcal{M}$ and $\mathcal{E}$ exhibit a scaling behavior of the form

$$
\begin{aligned}
& P_{L}(\mathcal{M})=A L^{\beta / \nu} P_{\mathcal{M}}^{*}\left(A L^{\beta / \nu} \delta \mathcal{M}\right), \\
& P_{L}(\mathcal{E})=B L^{(1-\alpha) / \nu} P_{\mathcal{E}}^{*}\left(B L^{(1-\alpha) / \nu} \delta \mathcal{E}\right)
\end{aligned}
$$

where $\alpha, \beta$, and $\nu$ are universal critical point exponents. the fixed point limiting operator distributions $P_{\mathcal{M}}^{*}$ and $P_{\mathcal{E}}^{*}$ are also universal for all systems of a given universality class. The quantities $\delta \mathcal{M}$ and $\delta \mathcal{E}$ measure deviations from criticality; $\delta \mathcal{M}=\mathcal{M}-\mathcal{M}_{c}, \quad \delta \mathcal{E}=\mathcal{E}-\mathcal{E}_{c} ; A$ and $B$ are systemspecific constants.

For the Ising universality class, the universal distribution functions mentioned above can be estimated from highresolution Monte Carlo simulations. ${ }^{17}$ To estimate the critical point of an Ising-class fluid, the temperature, chemical potential and the mixing parameters are tuned so that the re-
TABLE I. Temperatures and chemical potentials used for the simulation of the truncated Lennard-Jones fluid, where $T^{*}=k_{B} T / \varepsilon$, with $k_{B}$ being Boltzmann's constant.

\begin{tabular}{cccccc}
\hline \hline Replica & $T^{*}$ & $\beta \mu$ & Replica & $T^{*}$ & $\beta \mu$ \\
\hline 1 & 1.20 & -2.74 & 10 & 0.82 & -4.52 \\
2 & 1.17 & -2.83 & 11 & 0.79 & -4.76 \\
3 & 1.11 & -3.02 & 12 & 0.76 & -5.02 \\
4 & 1.07 & -3.18 & 13 & 0.73 & -5.30 \\
5 & 1.03 & -3.35 & 14 & 0.70 & -5.63 \\
6 & 0.99 & -3.51 & 15 & 0.67 & -5.99 \\
7 & 0.94 & -3.75 & 16 & 0.64 & -6.38 \\
8 & 0.90 & -3.98 & 17 & 0.62 & -6.66 \\
9 & 0.86 & -4.23 & 18 & 0.60 & -6.97 \\
\hline \hline
\end{tabular}

sulting distributions $P_{L}(\mathcal{M})$ and $P_{L}(\mathcal{E})$ collapse onto those of the Ising model. Values corresponding to the thermodynamic limit can be estimated by extrapolating finite-size values according to the scaling behavior

$$
\begin{aligned}
& T_{c}(L)-T_{c}(\infty) \propto L^{-(\theta+1) / \nu}, \\
& \rho_{c}(L)-\rho_{c}(\infty) \propto L^{-(1-\alpha) / \nu},
\end{aligned}
$$

where $\alpha, \theta$, and $\nu$ are universal exponents. For the 3D (three-dimensional) Ising universality class, $\alpha \approx 0.11, \theta$ $\approx 0.54, \beta \approx 0.312$, and $\nu \approx 0.629$.

\section{RESULTS AND DISCUSSION}

\section{A. Results for Lennard-Jones fluids}

In our calculations for Lennard-Jones fluids, we used 18 replicas, each having a box size $L=7 \sigma$. The temperature and chemical potential of each replica are reported in Table I. These values were selected to guarantee frequent swaps between replicas. Configuration swaps were attempted every 10 Monte Carlo steps; a total of $10^{6}$ Monte Carlo steps were performed to generate the energy and density histograms required to construct joint $(N, U)$ distributions for each replica.

During a simulation, we keep track of "physical" replicas as well as "logical" replicas. A physical replica is an actual collection of atoms that we follow throughout the course of the simulation. A logical replica is whatever configuration happens to visit a specific box at some specified conditions of temperature and chemical potential (e.g., box $i$, at $\mu_{i}$ and $T_{i}$ ). Figure 1 shows the evolution of a logical replica at $T^{*}=0.73$ and $\beta \mu=-5.30$, as a function of Monte Carlo steps; the ordinate axis indicates which physical replica happens to be visiting the logical replica at $T^{*}=0.73$ at any given time during the simulation. This serves to illustrate how configurations are swapped during the course of the simulation. As can be inferred from Fig. 1, a physical replica visits each logical replica relatively frequently and uniformly. After a successful configuration swap, two logical replicas adopt a completely new configuration. Further, configurations corresponding to logical replicas at low temperatures and high densities are "passed" over to logical replicas at higher temperatures and lower densities; such configurations can then relax more expeditiously and then be passed back to lower temperature replicas. This process greatly ac- 


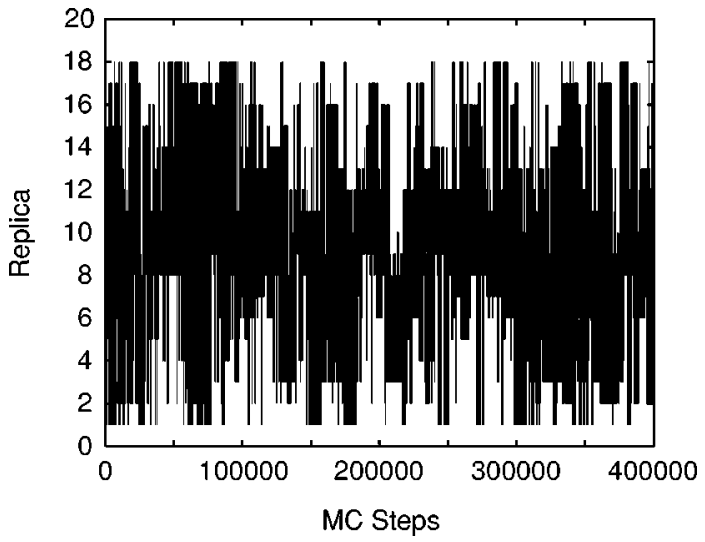

FIG. 1. Replica number as a function of Monte Carlo steps, for $T^{*}=0.73$ and $\beta \mu=-5.30$

celerates the relaxation of the global system and facilitates sampling of phase space under adverse conditions of temperature and chemical potential.

Figure 2 shows the probability density of the marginal distribution for the number of particles in the logical replica at $T^{*}=0.73$ and $\beta \mu=-5.30$. The chemical potential of that replica corresponds to a thermodynamic state slightly off the saturated liquid line. We would like to emphasize the occurrence of two distinct peaks in that density distribution. Given the fact that the temperature is well below the critical temperature, the observed "tunneling" behavior between a condensed phase and its vapor indicates that the large freeenergy barrier associated with the vapor-liquid phase transition can be overcome by the hyper-parallel tempering method. Such a crossing of high-energy barriers greatly facilitates the simulation of phase transitions. In contrast to a multicanonical sampling method, this tunneling is achieved by simple configuration swaps, rather than by artificial, trialand-error flattening of free energy barriers; intermediate samples in unstable regimes are not necessary in this algorithm.

To demonstrate how accurately the new method can sample the relative weights of the two peaks, we also show

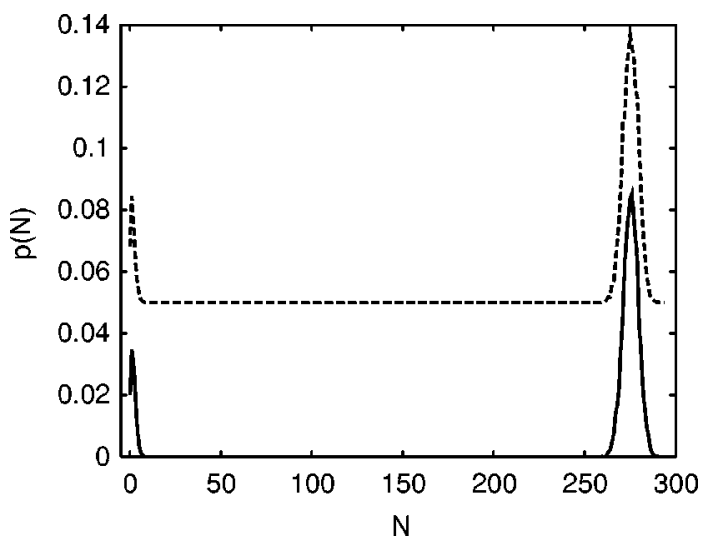

FIG. 2. Probability density of the marginal distribution of number of particles for $T^{*}=0.73, \beta \mu=-5.30$. The solid line represents the original histogram for the system at these conditions. The dashed line depicts the combined histogram that results from a full histogram reweighting analysis of all results at all conditions studied here (i.e., it is the "true" histogram).

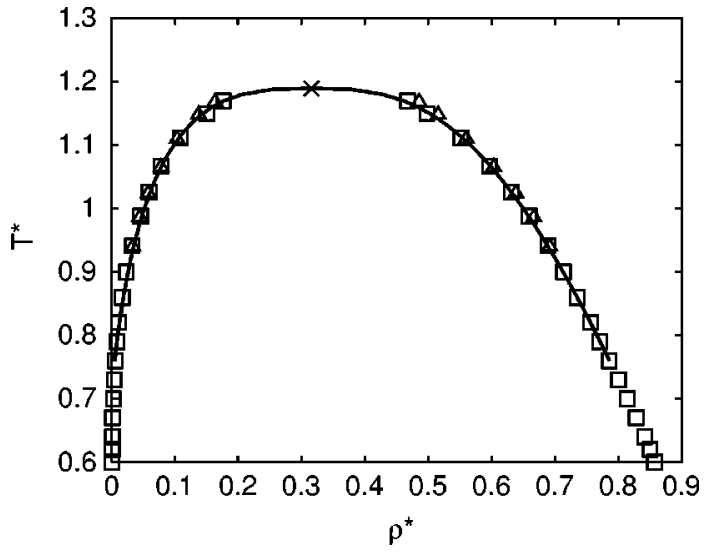

FIG. 3. Phase diagram (vapor-liquid equilibria) for a truncated LennardJones fluid. The squares correspond the results of this work, and the triangles show results reported by Wilding (Ref. 18). Statistical errors are smaller than the symbol size. The solid line is an Ising form fit to the simulation data.

in the figure the "real" density probability distribution corresponding the same thermodynamic conditions; the latter was calculated from histogram reweighting of data obtained from all 18 boxes. For clarity, that distribution is shifted by 0.05 .

Figure 3 shows the phase diagram calculated from histograms corresponding to all 18 logical replicas. Also shown are literature data for the same fluid. ${ }^{18}$ As expected from a correct algorithm, the agreement between the two sets of data is satisfactory. The slight discrepancies at high temperatures are due to different definitions of the equilibrium saturated density. In this work we regard the mean density corresponding to a peak of the distribution as the equilibrium value; Wilding defines it as the peak value of the distribution. As can be seen in Fig. 3, the proposed method is able to generate phase equilibrium data at temperatures and densities in the near vicinity of the triple point of the truncated LennardJones fluid (e.g., $T^{*}=0.60$ and $\rho^{*}=0.86$ ). A simple Gibbs ensemble method or conventional grand canonical simulations would be difficult and unreasonably demanding under such conditions.

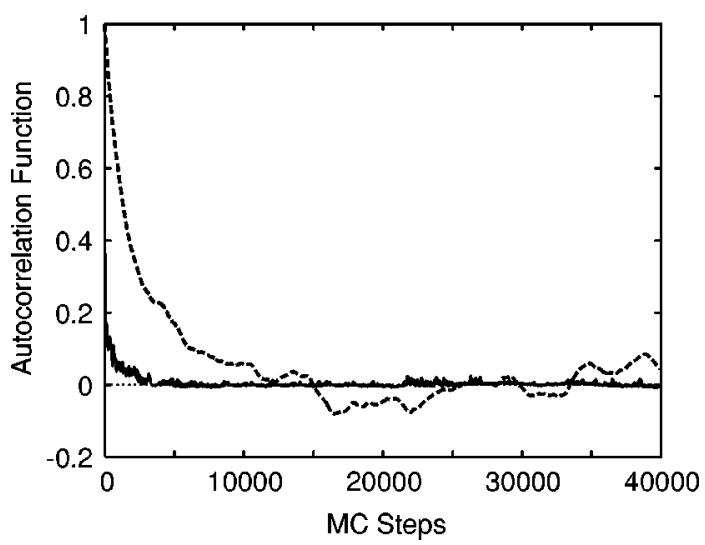

FIG. 4. Autocorrelation function for the average potential energy per atom for a Lennard-Jones fluid, corresponding to the lowest temperature replica ( $\left.T^{*}=0.60, \beta \mu=-6.97\right)$. The solid line corresponds to results of hyperparallel tempering simulations. The dashed line shows results from a conventional grand canonical Monte Carlo simulation. 
TABLE II. Temperatures and chemical potentials used for the simulation of the restricted primitive model $(L=13 \sigma)$, where $T^{*}=4 \pi D D_{0} \sigma k_{B} T / e^{2}$, where $D$ is the dielectric constant, $D_{0}$ the dielectric permeability of vacuum, $k_{B}$ Boltzmann's constant, and $e$ is the charge of the electron.

\begin{tabular}{clcccc}
\hline \hline Replica & \multicolumn{1}{c}{$T^{*}$} & $\beta \mu$ & Replica & $T^{*}$ & $\beta \mu$ \\
\hline 1 & 0.052 & -25.82 & 8 & 0.045 & -29.60 \\
2 & 0.050 & -26.79 & 9 & 0.044 & -30.25 \\
3 & 0.0492 & -27.19 & 10 & 0.043 & -30.92 \\
4 & 0.0485 & -27.55 & 11 & 0.042 & -31.66 \\
5 & 0.0475 & -28.10 & 12 & 0.041 & -32.43 \\
6 & 0.0468 & -28.50 & 13 & 0.040 & -33.24 \\
7 & 0.046 & -28.98 & & & \\
\hline \hline
\end{tabular}

As a measure of the efficiency of the new method, we show in Fig. 4 the autocorrelation function of the average potential energy per atom, evaluated at the lowest temperature replica studied here $\left(T^{*}=0.60\right.$ and $\left.\rho^{*}=0.86\right)$. As can be seen in the figure, for hyper-parallel tempering the energy autocorrelation function decays to zero after about 2000 Monte Carlo (MC) steps. For a conventional grand canonical simulation, it decays to zero after about 20000 steps. From an energy autocorrelation point of view, hyper-parallel tempering is about one order of magnitude more efficient than the conventional method. Note, however, that for simulations of phase transitions, a more appropriate measure of efficiency is provided by the "tunneling time," i.e., the time required to observe a jump from a vapor-like phase to a liquidlike phase during a simulation. A conventional method is unable to produce such jumps, at least not in a reasonable amount of time; hyper-parallel tempering routinely gives rise to such jumps. In this regard, the new method is considerably more efficient than existing algorithms for open ensembles: Difficult simulations that were not possible with previously available techniques could become feasible with HPTMC.

\section{B. Results for the restricted primitive model}

In this work, four different box sizes are used for the restricted primitive model: $L=13,15,16$, and $17 \sigma$. The length of the runs is about 1 to $2 \times 10^{6} \mathrm{MC}$ steps, depending

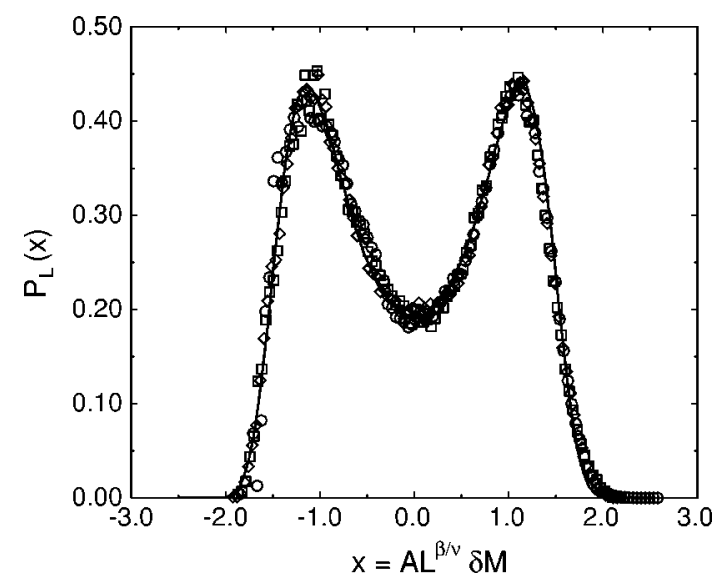

FIG. 5. Order parameter distributions for the restricted primitive model and their matching to the limiting Ising universal form. Circles: $L=13 \sigma$; Squares: $L=16 \sigma$; Diamonds: $L=17 \sigma$.

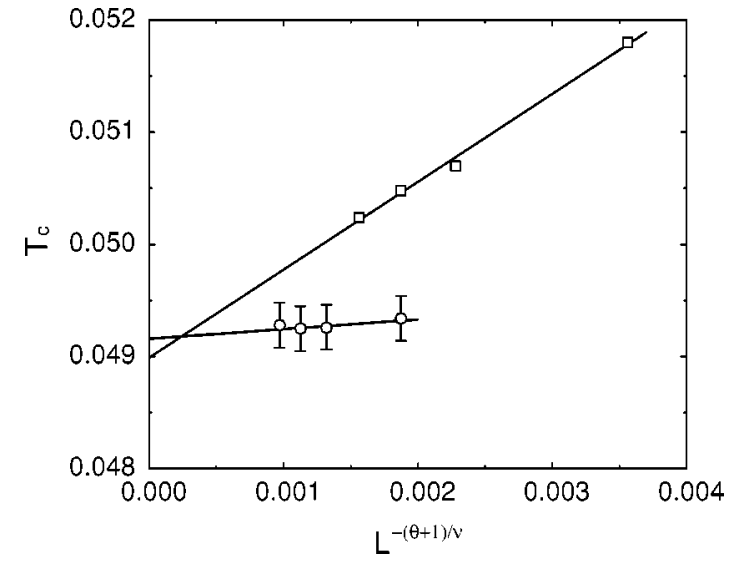

FIG. 6. Critical temperature scaling with system size for the restricted primitive model. The circles show results of this work, and the squares show data reported by Orkoulas et al. (1999); note that error bars were not reported by these authors.

on the box size. For the $L=13 \sigma$ box, 13 replicas are used to get the coexistence curve. For other box sizes, 3-6 replicas are used, mainly to explore the behavior of the model near the critical point. The coexistence curve near the critical point, of course, can also be obtained by these runs. Again, the values of temperature and chemical potential of these replicas are chosen close enough to each other so that configuration swaps can occur frequently. Also, they are chosen to be in the neighborhood of the coexistence curve. Table II reports the values for $L=13 \sigma$.

We estimated the critical point of the restricted primitive model by matching the order parameter distribution onto the Ising universal form. ${ }^{17}$ Figure 5 shows the result of such matching. As the runs are not too long (only 1 to $2 \times 10^{6} \mathrm{MC}$ steps), the quality of the matching is not perfect, but it is still satisfactory. We also observed that for the smaller boxes, there are not enough data points on the low-density side; for the largest box size, however, the situation is much better. The box-size dependence of the critical temperature and critical density is shown in Figs. 6 and 7, respectively. Our estimates of critical temperature and density for infinite box

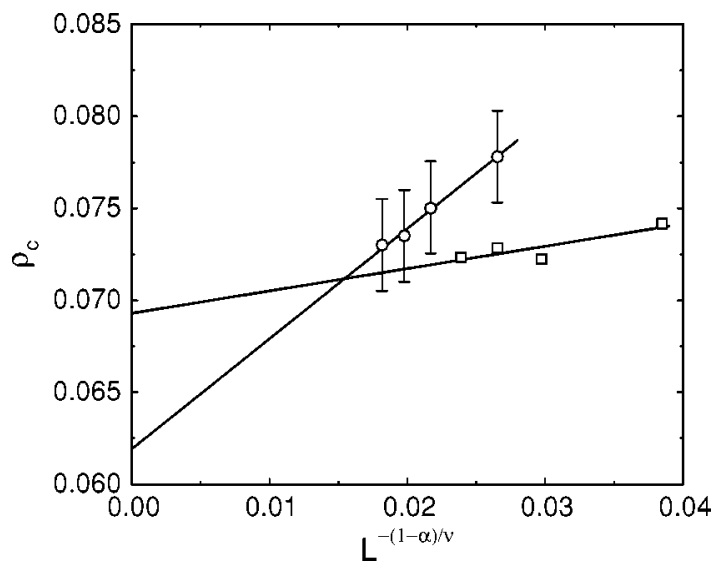

FIG. 7. Critical density scaling with system size for the restricted primitive model. The circles show results of this work, and the squares show data reported by Orkoulas et al. (1999); note that error bars were not reported by these authors. 
TABLE III. Critical point parameters in the limit of infinite system size.

\begin{tabular}{lcc}
\hline \hline \multicolumn{1}{c}{ Author } & $T_{c}^{*}$ & $\rho_{c}^{*}$ \\
\hline This work & $0.0492 \pm 0.0003$ & $0.062 \pm 0.005$ \\
Orkoulas et al. (1999) & $0.0490 \pm 0.0003$ & $0.070 \pm 0.005$ \\
Caillol et al. $(1997)$ & $0.0488 \pm 0.0002$ & $0.080 \pm 0.005$ \\
\hline \hline
\end{tabular}

size, along with those reported by earlier researchers, are summarized in Table III. Within the uncertainty of the calculations, our results are consistent with those reported by earlier researchers. ${ }^{23,24}$ Note, however, that our simulations and extrapolations to infinite size suggest that the precise value of $\rho_{c}^{*}$ is slightly lower than that reported in previous studies. In addition, Figs. 6 and 7 also show that the slopes of the linear relationship between the critical parameters and system size are quite different from those reported by Orkoulas et al. ${ }^{23}$

The coexistence curve of the restricted primitive model is shown in Fig. 8. Our results are compared with earlier data reported by Panagiotopoulos, ${ }^{25}$ Caillol, ${ }^{26}$ and Orkoulas and Panagiotopoulos. ${ }^{22,23}$ Our results exhibit small but systematic deviations from the latest data reported by Orkoulas and Panagiotopoulos. ${ }^{23}$ On the high-density side, our results are systematically smaller than the reported data, while on the low-density side, our results are slightly higher. At this point, the origin of such disagreement is uncertain. One possible origin could be that in our simulations, metal (conducting) boundary conditions are applied in the calculation of the Ewald sum, while in Orkoulas and Panagiotopoulos work, vacuum boundary conditions were employed. As mentioned earlier, it has been argued in the literature that conducting boundary conditions should be used for simulations of charged systems. The source of the discrepancy could also be that the simulation method employed here is more efficient than that of Orkoulas and Panagiotopoulos, and it therefore, permits simulations of larger systems with a smaller degree of correlation between successive configurations; our results

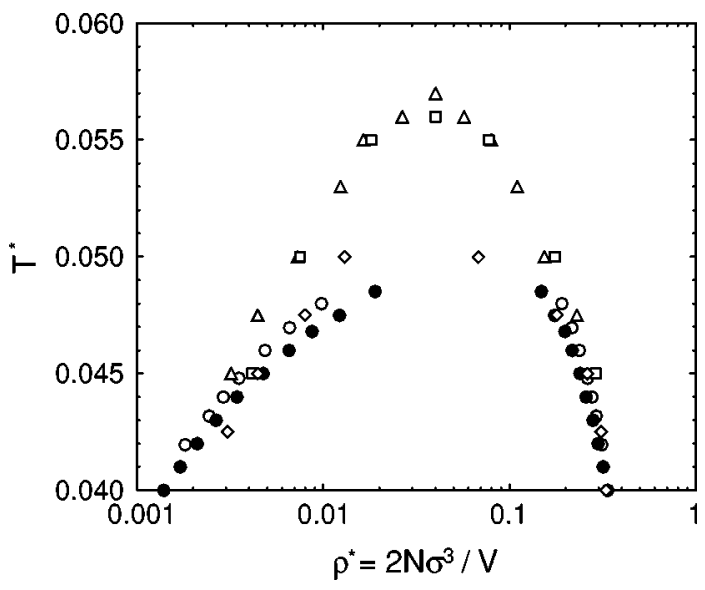

FIG. 8. Coexistence curve for the restricted primitive model. The filled circles show results of this work, the open circles show results reported by Orkoulas and Panagiotopoulos (1999), the squares are results reported by Panagiotopoulos (1992), the triangles show results reported by Caillol (1994), and the diamonds are results reported by Orkoulas and Panagiotopoulos (1994). correspond to systems that are generally much larger than those employed by Orkoulas and Panagiotopoulos. ${ }^{23}$

In that regard, we would like to draw special attention to the computational effort required for our simulations. As mentioned above, $10^{6}$ configurations were used to construct the coexistence curve. Multiplying by the number of boxes, a total of about $10^{7}$ configurations were generated. Orkoulas and Panagiotopoulos report that, to obtain results of comparable accuracy using a grand canonical method, about $10^{9}$ configurations are necessary. These numbers suggest that the new hyper-parallel tempering method is about two orders of magnitude faster than grand canonical ensemble simulations of comparable systems.

\section{CONCLUSIONS}

In this work, we have presented a new generalized hyper-parallel tempering Monte Carlo simulation method. The method has been shown to be superior to conventional simulation techniques for the study of phase transitions in fluids. The method has the added benefit of being remarkably simple to implement. While some preliminary trial and error runs is necessary to optimize the performance, we found that this could be done with relative ease. We demonstrated some of its advantages by applying it to the simulation of a simple truncated Lennard-Jones fluid, down to temperatures close to the triple point, and to the simulation of the restricted primitive model. Our results for the Lennard-Jones fluid are completely consistent with the literature. Our results for the PRM exhibit small but systematic deviations from those reported by previous authors for smaller systems. Our results also show that the new method is capable of overcoming the large free energy barrier associated with a vapor-liquid or liquidliquid phase transition, and that it is also capable of relaxing the system much faster than traditional grand canonical ensemble simulations.

One disadvantage of the method is that a good set of temperatures and chemical potentials must be chosen in order to maximize the efficiency of the method. This can be achieved by a few short test runs in small systems. Two major considerations must be taken into account for selection of appropriate simulation conditions:

(1) For simulation of coexistence, the state points should be near the coexistence curve. For lower temperatures, it is better to be slightly off to the liquid side; since the vapor density is very small at low temperatures, simulations at such low densities usually do not provide much information.

(2) The state points of neighboring replicas must be close to each other, so that enough configuration swaps can occur. As the box size becomes large, state points must be closer; this is a reflection of the fact that the relevant distribution functions become narrower as the system gets larger.

The first consideration is not unique to HPTMC, but it occurs whenever $\mu V T$ and histogram reweighting techniques are employed. Fortunately, the chemical potential can be manipulated with relative ease, particularly for small systems, 
to explore the phase behavior approximately. Our experience suggests that this preliminary search of phase space requires only a small fraction of the overall computer time requirements.

The second consideration also arises whenever multihistogram reweighting techniques are employed to analyze data from a series of simulations. Unless the degree of overlap between histograms is sufficiently large, multihistogram reweighting techniques are not useful for calculating phase diagrams. By construction, if two histograms overlap sufficiently, the HPTMC method gives rise to a reasonable acceptance rate for swap trial moves. One could therefore argue that if a histogram reweighting technique is to be used, HPTMC does not incur in additional computational requirements and it improves efficiency significantly. In this work, our selection of state points led to an acceptance rate of $\sim 20 \%-30 \%$ for configuration swap attempts. Note, however, that at these preliminary stages of our work we have not made any attempts to optimize the swapping rate and examine its effects on overall efficiency.

\section{ACKNOWLEDGMENTS}

This work was supported through a PECASE award from the National Science Foundation to J.J.dP and through award CTS-9901430. J.J.dP. is also grateful to the Dreyfus Foundation for a Teacher-Scholar Award. J.J.dP. wishes to acknowledge the hospitality extended to him by Gustavo Chapela during a sabbatical stay at the Mexican Petroleum Institute.
${ }^{1}$ B. Berg and T. Neuhaus, Phys. Lett. B 267, 249 (1991).

${ }^{2}$ B. Berg and T. Neuhaus, Phys. Rev. Lett. 68, 9 (1992).

${ }^{3}$ B. Hessebo and R. B. Stinchcombe, Phys. Rev. Lett. 74, 2151 (1995).

${ }^{4}$ A. P. Lyubartsev, A. A. Martinovski, S. V. Shevkunov, and P. N. Vorontsov-Velyaminov, J. Chem. Phys. 96, 1776 (1992).

${ }^{5}$ E. Marinari and G. Parisi, Europhys. Lett. 19, 451 (1992).

${ }^{6}$ F. A. Escobedo and J. J. de Pablo, J. Chem. Phys. 105, 4391 (1996).

${ }^{7}$ D. D. Frantz, D. L. Freeman, and J. D. Doll, J. Chem. Phys. 93, 2769 (1990).

${ }^{8}$ W. Ortiz, A. Perlloni, and G. E. Lopez, Chem. Phys. Lett. 298, 66 (1998).

${ }^{9}$ M. C. Tesi, E. J. Janse van Rensburg, E. Orlandini, and S. G. Whittington, J. Stat. Phys. 82, 155 (1996).

${ }^{10}$ U. H. E. Hansmann, Chem. Phys. Lett. 281, 140 (1997).

${ }^{11}$ M. G. Wu and M. W. Deem, Mol. Phys. 97, 559 (1999).

${ }^{12}$ A. M. Ferrenberg and R. H. Swendsen, Phys. Rev. Lett. 61, 2635 (1988).

${ }^{13}$ A. M. Ferrenberg and R. H. Swendsen, Phys. Rev. Lett. 63, 1195 (1989).

${ }^{14}$ A. M. Ferrenberg and R. H. Swendsen, Comput. Phys. Sept/Oct, 101 (1989).

${ }^{15}$ A. D. Bruce and N. B. Wilding, Phys. Rev. Lett. 68, 193 (1992).

${ }^{16}$ N. B. Wilding and A. D. Bruce, J. Phys.: Condens. Matter 4, 3087 (1992).

${ }^{17}$ N. B. Wilding and M. Müller, J. Chem. Phys. 102, 2562 (1995).

${ }^{18}$ N. B. Wilding, Phys. Rev. E 52, 602 (1995).

${ }^{19}$ T. L. Hill, Thermodynamics of Small Systems (Dover, New York, 1962).

${ }^{20}$ D. Frenkel and B. Smit, Understanding Molecular Simulation - From Algorithms to Applications (Academic, San Diego, 1996).

${ }^{21}$ M. Neumann, Mol. Phys. 50, 841 (1983).

${ }^{22}$ G. Orkoulas and A. Z. Panagiotopoulos, J. Chem. Phys. 101, 1452 (1994).

${ }^{23}$ G. Orkoulas and A. Z. Panagiotopoulos, J. Chem. Phys. 110, 1581 (1999).

${ }^{24}$ J. M. Caillol, D. Levesque, and J. J. Weis, Phys. Rev. Lett. 77, 4039 (1996); J. Chem. Phys. 107, 1565 (1997).

${ }^{25}$ A. Z. Panagiotopoulos, Fluid Phase Equilibria 76, 97 (1992).

${ }^{26}$ J. M. Caillol, J. Chem. Phys. 100, 2161 (1994).

${ }^{27}$ Q. Yan and J. J. de Pablo (unpublished). 